\title{
Space-Time Block Coding for Wireless Communications: Performance Results
}

\author{
Vahid Tarokh, Member, IEEE, Hamid Jafarkhani, Member, IEEE, and A. Robert Calderbank, Fellow, IEEE
}

\begin{abstract}
We document the performance of space-time block codes [13], [14], which provide a new paradigm for transmission over Rayleigh fading channels using multiple transmit antennas. Data is encoded using a space-time block code, and the encoded data is split into $n$ streams which are simultaneously transmitted using $n$ transmit antennas. The received signal at each receive antenna is a linear superposition of the $n$ transmitted signals perturbed by noise. Maximum likelihood decoding is achieved in a simple way through decoupling of the signals transmitted from different antennas rather than joint detection. This uses the orthogonal structure of the space-time block code and gives a maximum likelihood decoding algorithm which is based only on linear processing at the receiver. We review the encoding and decoding algorithms for various codes and provide simulation results demonstrating their performance. It is shown that using multiple transmit antennas and space-time block coding provides remarkable performance at the expense of almost no extra processing.
\end{abstract}

Index Terms - Diversity, multipath channels, multiple antennas, space-time codes, wireless communication.

\section{INTRODUCTION}

$\mathbf{I}$ N MOST situations, the wireless channel suffers attenuation due to destructive addition of multipaths in the propagation media and to interference from other users. The channel statistic is significantly often Rayleigh which makes it difficult for the receiver to reliably determine the transmitted signal unless some less attenuated replica of the signal is provided to the receiver. This technique is called diversity, which can be provided using temporal, frequency, polarization, and spatial resources [3], [6], [7]-[9], [11], [16], [17]. In many situations, however, the wireless channel is neither significantly timevariant nor highly frequency selective. This forces the system engineers to consider the possibility of deploying multiple antennas at both the transmitter and receiver to achieve spatial diversity.

Only recently has transmit diversity been studied extensively as a method of combating detrimental effects in wireless fading channels because of its relative simplicity of implementation and feasibility of having multiple antennas at the base station. The first bandwidth efficient transmit diversity scheme was proposed by Wittneben [18], and it includes the delay diversity scheme of Seshadri and Winters [10] as a special

Manuscript received May 13, 1998; revised August 28, 1998 and October 12, 1998.

V. Tarokh and A. R. Calderbank are with AT\&T Labs-Research, Florham Park, NJ 07932 USA.

H. Jafarkhani is with AT\&T Labs-Research, Red Bank, NJ 07701 USA. Publisher Item Identifier S 0733-8716(99)02465-8. case. Later Foschini introduced a multilayered space-time architecture [4].

More recently, space-time trellis coding has been proposed [12] which combines signal processing at the receiver with coding techniques appropriate to multiple transmit antennas and provides significant gain over [10] and [18]. Specific space-time trellis codes designed for two-four transmit antennas perform extremely well in slow fading environments (typical of indoor transmission) and come within 2-3 dB of the outage capacity computed by Telatar [15] and independently by Foschini and Gans [5]. The bandwidth efficiency is about three-four times that of current systems. The space-time codes presented in [12] provide the best possible tradeoff between constellation size, data rate, diversity advantage, and trellis complexity. When the number of transmit antennas is fixed, the decoding complexity of space-time trellis coding (measured by the number of trellis states in the decoder) increases exponentially as a function of both the diversity level and the transmission rate.

In addressing the issue of decoding complexity, Alamouti discovered a remarkable scheme for transmission using two transmit antennas [1]. Space-time block coding, introduced in [13] and [14], generalizes the transmission scheme discovered by Alamouti to an arbitrary number of transmit antennas and is able to achieve the full diversity promised by the transmit and receive antennas. These codes retain the property of having a very simple maximum likelihood decoding algorithm based only on linear processing at the receiver [13], [14]. For real signal constellations (such as PAM), they provide the maximum possible transmission rate allowed by the theory of space-time coding [12]. For complex constellations, space-time block codes can be constructed for any number of transmit antennas, and again these codes have remarkably simple decoding algorithms based only on linear processing at the receiver. They provide full spatial diversity and half of the maximum possible transmission rate allowed by the theory of space-time coding. For complex constellations and for the specific cases of three and four transmit antennas, these diversity schemes were improved to provide 3/4 of the maximum possible transmission rate [13], [14].

The purpose of this paper is to evaluate the performance of the space-time block codes constructed in [13] and [14] and to provide the details of the encoding and decoding procedures. We begin by considering encoding and decoding algorithms for some of these codes. We then provide simulation results confirming that with space-time block coding and 
multiple transmit antennas, a significant performance gain can be achieved at almost no processing expense.

The outline of this paper is as follows. In Section II, we provide a mathematical model for the multiple antenna communication systems. We review examples of space-time block codes constructed in [13] and [14] and will describe their encoding and decoding algorithms in Section III. Section IV analyzes the performance of the space-time block code and shows that our simple decoding scheme achieves exactly the same performance as receive maximum ratio combining. Section V provides simulation results demonstrating the performance of these codes. Finally, Section VI presents our conclusions and final comments.

\section{THE TRANSMISSION MODEL}

We consider a wireless communication system with $n$ antennas at the base station and $m$ antennas at the remote. At each time slot $t$, signals $c_{t}^{i}, i=1,2, \ldots, n$ are transmitted simultaneously from the $n$ transmit antennas. The channel is assumed to be a flat fading channel and the path gain from transmit antenna $i$ to receive antenna $j$ is defined to be $\alpha_{i, j}$. The path gains are modeled as samples of independent complex Gaussian random variables with variance 0.5 per real dimension. This assumption can be relaxed without any change to the method of encoding and decoding [12]. The wireless channel is assumed to be quasi-static so that the path gains are constant over a frame of length $l$ and vary from one frame to another.

At time $t$ the signal $r_{t}^{j}$, received at antenna $j$, is given by

$$
r_{t}^{j}=\sum_{i=1}^{n} \alpha_{i, j} c_{t}^{i}+\eta_{t}^{j}
$$

where the noise samples $\eta_{t}^{j}$ are independent samples of a zero-mean complex Gaussian random variable with variance $n /(2 \mathrm{SNR})$ per complex dimension. The average energy of the symbols transmitted from each antenna is normalized to be one, so that the average power of the received signal at each receive antenna is $n$ and the signal-to-noise ratio is SNR.

Assuming perfect channel state information is available, the receiver computes the decision metric

$$
\sum_{t=1}^{l} \sum_{j=1}^{m}\left|r_{t}^{j}-\sum_{i=1}^{n} \alpha_{i, j} c_{t}^{i}\right|^{2}
$$

over all code words

$$
c_{1}^{1} c_{1}^{2} \cdots c_{1}^{n} c_{2}^{1} c_{2}^{2} \cdots c_{2}^{n} \cdots \cdots c_{l}^{1} c_{l}^{2} \cdots c_{l}^{n}
$$

and decides in favor of the code word that minimizes the sum.

\section{Space-Time Block CodeS}

\section{A. Encoding Algorithm}

A space-time block code is defined by a $p \times n$ transmission matrix $\mathcal{G}$. The entries of the matrix $\mathcal{G}$ are linear combinations of the variables $x_{1}, x_{2}, \ldots, x_{k}$ and their conjugates. The number of transmission antennas is $n$, and we usually use it to separate different codes from each other. For example, $\mathcal{G}_{2}$ represents a code which utilizes two transmit antennas and is defined by

$$
\mathcal{G}_{2}=\left(\begin{array}{rr}
x_{1} & x_{2} \\
-x_{2}^{*} & x_{1}^{*}
\end{array}\right) .
$$

We assume that transmission at the baseband employs a signal constellation $\mathcal{A}$ with $2^{b}$ elements. At time slot $1, k b$ bits arrive at the encoder and select constellation signals $s_{1}, \ldots, s_{k}$. Setting $x_{i}=s_{i}$ for $i=1,2, \ldots, k$ in $\mathcal{G}$, we arrive at a matrix $\mathcal{C}$ with entries linear combinations of $s_{1}, s_{2}, \ldots, s_{k}$ and their conjugates. So, while $\mathcal{G}$ contains indeterminates $x_{1}, x_{2}, \ldots, x_{k}, \mathcal{C}$ contains specific constellation symbols (or their linear combinations) which are transmitted from $n$ antennas for each $k b$ bits as follows. If $c_{t}^{i}$ represents the element in the $t$ th row and the $i$ th column of $\mathcal{C}$, the entries $c_{t}^{i}, i=1,2, \ldots, n$ are transmitted simultaneously from transmit antennas $1,2, \ldots, n$ at each time slot $t=$ $1,2, \ldots, p$. So, the $i$ th column of $\mathcal{C}$ represents the transmitted symbols from the $i$ th antenna and the $t$ th row of $\mathcal{C}$ represents the transmitted symbols at time slot $t$. Note that $\mathcal{C}$ is basically defined using $\mathcal{G}$, and the orthogonality of $\mathcal{G}$ 's columns allows a simple decoding scheme which will be explained in the sequel. Since $p$ time slots are used to transmit $k$ symbols, we define the rate $R$ of the code to be $R=k / p$. For example, the rate of $\mathcal{G}_{2}$ is one.

In this work, we consider the performance of the following rate half space-time block codes:

$$
\mathcal{G}_{3}=\left(\begin{array}{rrr}
x_{1} & x_{2} & x_{3} \\
-x_{2} & x_{1} & -x_{4} \\
-x_{3} & x_{4} & x_{1} \\
-x_{4} & -x_{3} & x_{2} \\
x_{1}^{*} & x_{2}^{*} & x_{3}^{*} \\
-x_{2}^{*} & x_{1}^{*} & -x_{4}^{*} \\
-x_{3}^{*} & x_{4}^{*} & x_{1}^{*} \\
-x_{4}^{*} & -x_{3}^{*} & x_{2}^{*}
\end{array}\right)
$$

and

$$
\mathcal{G}_{4}=\left(\begin{array}{rrrr}
x_{1} & x_{2} & x_{3} & x_{4} \\
-x_{2} & x_{1} & -x_{4} & x_{3} \\
-x_{3} & x_{4} & x_{1} & -x_{2} \\
-x_{4} & -x_{3} & x_{2} & x_{1} \\
x_{1}^{*} & x_{2}^{*} & x_{3}^{*} & x_{4}^{*} \\
-x_{2}^{*} & x_{1}^{*} & -x_{4}^{*} & x_{3}^{*} \\
-x_{3}^{*} & x_{4}^{*} & x_{1}^{*} & -x_{2}^{*} \\
-x_{4}^{*} & -x_{3}^{*} & x_{2}^{*} & x_{1}^{*}
\end{array}\right) .
$$

We also consider the rate $3 / 4$ codes shown in (6) and (7), found at the bottom of the next page, for three and four transmit antennas (we use $\mathcal{H}$ instead of $\mathcal{G}$ to distinguish these codes). Next we review the decoding of these codes.

\section{B. The Decoding Algorithm}

Maximum likelihood decoding of any space-time block code can be achieved using only linear processing at the receiver, and we illustrate this by some examples. 
The space-time block code $\mathcal{G}_{2}$ (first proposed by [1]) uses the transmission matrix in (3). Suppose that there are $2^{b}$ signals in the constellation. At the first time slot $2 b$ bits arrive at the encoder and select two complex symbols $s_{1}$ and $s_{2}$. These symbols are transmitted simultaneously from antennas one and two, respectively. At the second time slot, signals $-s_{2}^{*}$ and $s_{1}^{*}$ are transmitted simultaneously from antennas one and two, respectively.

Then maximum likelihood detection amounts to minimizing the decision metric

$$
\sum_{j=1}^{m}\left(\left|r_{1}^{j}-\alpha_{1, j} s_{1}-\alpha_{2, j} s_{2}\right|^{2}+\left|r_{2}^{j}+\alpha_{1, j} s_{2}^{*}-\alpha_{2, j} s_{1}^{*}\right|^{2}\right)
$$

over all possible values of $s_{1}$ and $s_{2}$. Note that due to the quasi-static nature of the channel, the path gains are constant over two transmissions. The minimizing values are the receiver estimates of $s_{1}$ and $s_{2}$, respectively. We expand the above metric and delete the terms that are independent of the codewords and observe that the above minimization is equivalent to minimizing

$$
\begin{aligned}
& -\sum_{j=1}^{m}\left[r_{1}^{j} \alpha_{1, j}^{*} s_{1}^{*}+\left(r_{1}^{j}\right)^{*} \alpha_{1, j} s_{1}+r_{1}^{j} \alpha_{2, j}^{*} s_{2}^{*}+\left(r_{1}^{j}\right)^{*} \alpha_{2, j} s_{2}\right. \\
& \left.-r_{2}^{j} \alpha_{1, j}^{*} s_{2}-\left(r_{2}^{j}\right)^{*} \alpha_{1, j} s_{2}^{*}+r_{2}^{j} \alpha_{2, j}^{*} s_{1}+\left(r_{2}^{j}\right)^{*} \alpha_{2, j} s_{1}^{*}\right] \\
& \quad+\left(\left|s_{1}\right|^{2}+\left|s_{2}\right|^{2}\right) \sum_{j=1}^{m} \sum_{i=1}^{2}\left|\alpha_{i, j}\right|^{2} .
\end{aligned}
$$

The above metric decomposes into two parts, one of which

$$
\begin{aligned}
& -\sum_{j=1}^{m}\left[r_{1}^{j} \alpha_{1, j}^{*} s_{1}^{*}+\left(r_{1}^{j}\right)^{*} \alpha_{1, j} s_{1}+r_{2}^{j} \alpha_{2, j}^{*} s_{1}+\left(r_{2}^{j}\right)^{*} \alpha_{2, j} s_{1}^{*}\right] \\
& \quad+\left|s_{1}\right|^{2} \sum_{j=1}^{m} \sum_{i=1}^{2}\left|\alpha_{i, j}\right|^{2}
\end{aligned}
$$

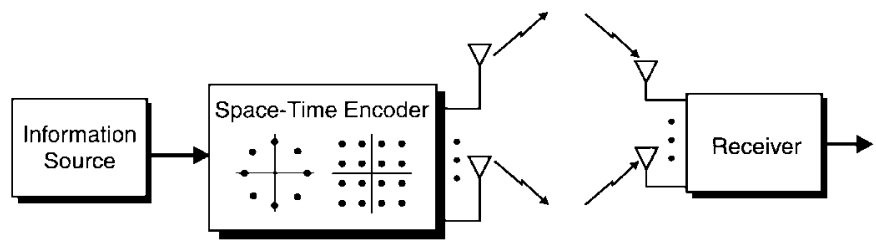

Fig. 1. System block diagram.

is only a function of $s_{1}$, and the other one

$$
\begin{aligned}
& -\sum_{j=1}^{m}\left[r_{2}^{j} \alpha_{2, j}^{*} s_{2}^{*}+\left(r_{1}^{j}\right)^{*} \alpha_{2, j} s_{2}-r_{2}^{j} \alpha_{1, j}^{*} s_{2}-\left(r_{2}^{j}\right)^{*} \alpha_{1, j} s_{2}^{*}\right] \\
& +\left|s_{2}\right|^{2} \sum_{j=1}^{m} \sum_{i=1}^{2}\left|\alpha_{i, j}\right|^{2}
\end{aligned}
$$

is only a function of $s_{2}$. Thus the minimization of (8) is equivalent to minimizing these two parts separately. This in turn is equivalent to minimizing the decision metric

$$
\begin{gathered}
\left|\left[\sum_{j=1}^{m}\left(r_{1}^{j} \alpha_{1, j}^{*}+\left(r_{2}^{j}\right)^{*} \alpha_{2, j}\right)\right]-s_{1}\right|^{2} \\
+\left(-1+\sum_{j=1}^{m} \sum_{i=1}^{2}\left|\alpha_{i, j}\right|^{2}\right)\left|s_{1}\right|^{2}
\end{gathered}
$$

for detecting $s_{1}$ and the decision metric

$$
\begin{gathered}
\left|\left[\sum_{j=1}^{m}\left(r_{1}^{j} \alpha_{2, j}^{*}-\left(r_{2}^{j}\right)^{*} \alpha_{1, j}\right)\right]-s_{2}\right|^{2} \\
+\left(-1+\sum_{j=1}^{m} \sum_{i=1}^{2}\left|\alpha_{i, j}\right|^{2}\right)\left|s_{2}\right|^{2}
\end{gathered}
$$

for decoding $s_{2}$. This is the simple decoding scheme described in [1], and there is no performance sacrifice for using it.

Similarly, the decoders for $\mathcal{G}_{3}, \mathcal{G}_{4}, \mathcal{H}_{3}$, and $\mathcal{H}_{4}$ can be derived. We provide the details of these decoders in the Appendix for the sake of presentation.

$$
\begin{aligned}
& \mathcal{H}_{3}=\left.\begin{array}{ccc}
x_{1} & x_{2} & \frac{x_{3}}{\sqrt{2}} \\
-x_{2}^{*} & x_{1}^{*} & \frac{x_{3}}{\sqrt{2}} \\
\frac{x_{3}^{*}}{\sqrt{2}} & \frac{x_{3}^{*}}{\sqrt{2}} & \frac{\left(-x_{1}-x_{1}^{*}+x_{2}-x_{2}^{*}\right)}{2} \\
\frac{x_{3}^{*}}{\sqrt{2}} & -\frac{x_{3}^{*}}{\sqrt{2}} & \frac{\left(x_{2}+x_{2}^{*}+x_{1}-x_{1}^{*}\right)}{2}
\end{array}\right) \\
& \mathcal{H}_{4}=\left(\begin{array}{cccc}
x_{1} & x_{2} & \frac{x_{3}}{\sqrt{2}} & \frac{x_{3}}{\sqrt{2}} \\
-x_{2}^{*} & x_{1}^{*} & \frac{x_{3}}{\sqrt{2}} & -\frac{x_{3}}{\sqrt{2}} \\
\frac{x_{3}^{*}}{\sqrt{2}} & \frac{x_{3}^{*}}{\sqrt{2}} & \frac{\left(-x_{1}-x_{1}^{*}+x_{2}-x_{2}^{*}\right)}{2} & -\frac{\left(x_{1}+x_{1}^{*}+x_{1}-x_{1}-x_{2}^{*}\right)}{2} \\
\frac{x_{3}^{*}}{\sqrt{2}} & -\frac{x_{3}^{*}}{\sqrt{2}} & \frac{\left(x_{2}+x_{2}^{*}+x_{1}-x_{1}^{*}\right)}{2} &
\end{array}\right)
\end{aligned}
$$




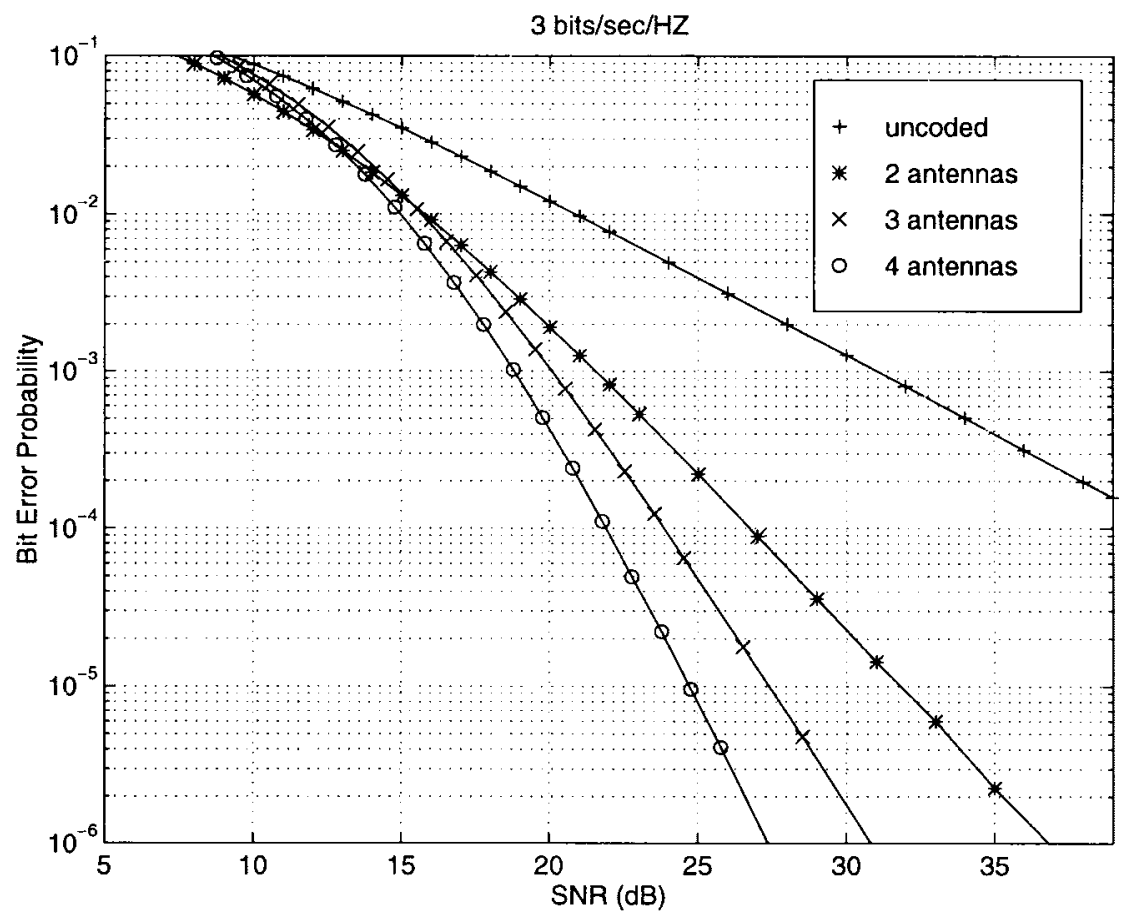

Fig. 2. Bit error probability versus SNR for space-time block codes at $3 \mathrm{bits} / \mathrm{s} / \mathrm{Hz}$; one receive antenna.

\section{PeRformance Analysis}

In this section, we analyze the performance of $\mathcal{G}_{4}$ when the energy of different symbols are equal to each other [e.g., phase-shift keying (PSK)]. Similar results can be achieved for other codes presented in Section III. The decoder minimizes the decision metric

$$
\begin{aligned}
& \mid\left[\sum _ { j = 1 } ^ { m } \left(r_{1}^{j} \alpha_{1, j}^{*}+r_{2}^{j} \alpha_{2, j}^{*}+r_{3}^{j} \alpha_{3, j}^{*}+r_{4}^{j} \alpha_{4, j}^{*}+\left(r_{5}^{j}\right)^{*} \alpha_{1, j}\right.\right. \\
& \left.\left.\quad+\left(r_{6}^{j}\right)^{*} \alpha_{2, j}+\left(r_{7}^{j}\right)^{*} \alpha_{3, j}+\left(r_{8}^{j}\right)^{*} \alpha_{4, j}\right)\right]-\left.s_{1}\right|^{2}
\end{aligned}
$$

for decoding $s_{1}$ which can be rewritten as $\left|\hat{s}_{1}-s_{1}\right|^{2}$ where

$$
\begin{aligned}
\hat{s}_{1}= & \sum_{j=1}^{m}\left(r_{1}^{j} \alpha_{1, j}^{*}+r_{2}^{j} \alpha_{2, j}^{*}+r_{3}^{j} \alpha_{3, j}^{*}+r_{4}^{j} \alpha_{4, j}^{*}\right. \\
& \left.+\left(r_{\tilde{5}}^{j}\right)^{*} \alpha_{1, j}+\left(r_{6}^{j}\right)^{*} \alpha_{2, j}+\left(r_{7}^{j}\right)^{*} \alpha_{3, j}+\left(r_{8}^{j}\right)^{*} \alpha_{4, j}\right) .
\end{aligned}
$$

By replacing $r_{t}^{j}$ from (1) in the above equation and simple manipulations, we arrive at

$$
\hat{s}_{1}=2 \sum_{j=1}^{m} \sum_{i=1}^{4}\left|\alpha_{i, j}\right|^{2} s_{1}+\Xi_{1}
$$

where

$$
\begin{aligned}
\Xi_{1}= & \sum_{j=1}^{m}\left(\eta_{1}^{j} \alpha_{1, j}^{*}+\eta_{2}^{j} \alpha_{2, j}^{*}+\eta_{3}^{j} \alpha_{3, j}^{*}+\eta_{4}^{j} \alpha_{4, j}^{*}\right. \\
& \left.+\left(\eta_{5}^{j}\right)^{*} \alpha_{1, j}+\left(\eta_{6}^{j}\right)^{*} \alpha_{2, j}+\left(\eta_{7}^{j}\right)^{*} \alpha_{3, j}+\left(\eta_{8}^{j}\right)^{*} \alpha_{4, j}\right) .
\end{aligned}
$$

By the last equation, it is immediate that given $\alpha_{i, j}, i=$ $1,2,3,4, j=1,2, \ldots, m$, the random variable $\Xi_{1}$ is a zero-mean complex Gaussian random variable with vari- ance $4 / \mathrm{SNR} \sum_{j=1}^{m} \sum_{i=1}^{4}\left|\alpha_{i, j}\right|^{2}$ per real dimension. Given $\alpha_{i, j}, i=1,2,3,4, j=1,2, \ldots, m$, the power of signal in $\hat{s}_{1}$ is $4\left[\sum_{j=1}^{m} \sum_{i=1}^{4}\left|\alpha_{i, j}\right|^{2}\right]^{2}$.

Next consider another communication scenario where $s_{1}$ is sent from one transmit antenna and is received using $4 m$ receive antennas. Suppose that the path gains from the transmit antenna to the receive antennas are $\alpha_{i, j}, i=1,2,3,4, j=$ $1,2, \ldots, m$. Given these path gains and following maximum ratio combining, the decision metric is of the form

$$
\tilde{s}_{1}=\sum_{j=1}^{m} \sum_{i=1}^{4}\left|\alpha_{i, j}\right|^{2} s_{1}+\Xi_{1}^{\prime}
$$

where the random variable $\Xi_{1}^{\prime}$ is a zero-mean complex Gaussian random variable with variance $1 / \mathrm{SNR} \sum_{j=1}^{m} \sum_{i=1}^{4}\left|\alpha_{i, j}\right|^{2}$ per real dimension. This is the same as (9) but both the power of signal and noise are reduced by a factor of $1 / 4$. It is clear that such a normalization does not affect the performance. Thus, it is concluded that using four transmit and $m$ receive antennas, the code $\mathcal{G}_{4}$ provides exactly the same performance as $4 m$ level receive maximum ratio combining.

\section{Simulation Results}

In this section, we provide simulation results for the performance of the codes given in the previous sections. Fig. 1 illustrates a block diagram of the system. The information source is encoded using a space-time block code, and the constellation symbols are transmitted from different antennas. The receiver estimates the transmitted bits by using the signals of the received antennas. Figs. 2 and 3 show bit and symbol error rates, respectively, for transmission of $3 \mathrm{bits} / \mathrm{s} / \mathrm{Hz}$. The results are reported for an uncoded 8-PSK and our space-time block codes using two, three, and four transmit antennas. Simulation results in Figs. 2 and 3 are given for one receive antenna. 


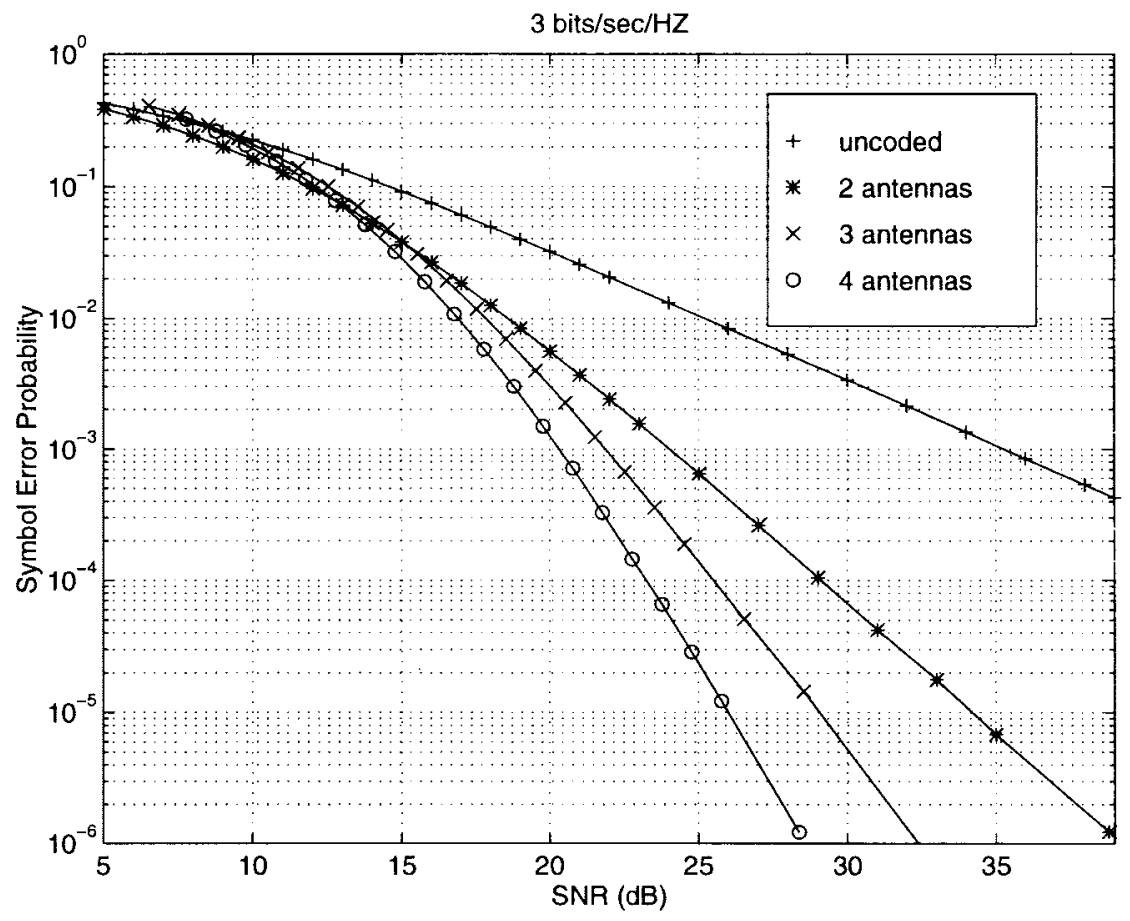

Fig. 3. Symbol error probability versus SNR for space-time block codes at $3 \mathrm{bits} / \mathrm{s} / \mathrm{Hz}$; one receive antenna.

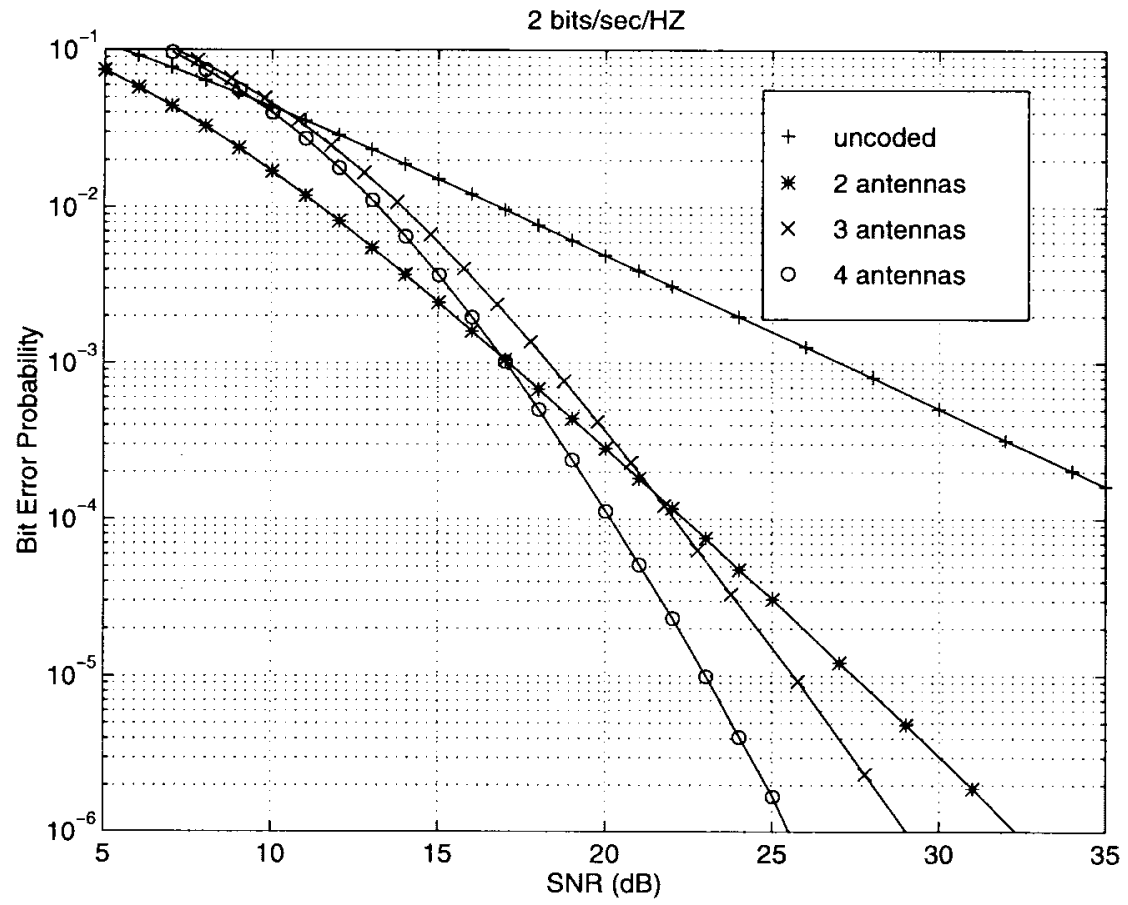

Fig. 4. Bit error probability versus SNR for space-time block codes at 2 bits/s/Hz; one receive antenna.

The transmission using two transmit antennas employs the 8PSK constellation and the code $\mathcal{G}_{2}$. For three and four transmit antennas, the 16-QAM constellation and the codes $\mathcal{H}_{3}$ and $\mathcal{H}_{4}$, respectively, are used. Since $\mathcal{H}_{3}$ and $\mathcal{H}_{4}$ are rate $3 / 4$ codes, the total transmission rate in each case is $3 \mathrm{bits} / \mathrm{s} / \mathrm{Hz}$. It is seen that at the bit error rate of $10^{-5}$ the rate $3 / 4$ 16-QAM code $\mathcal{H}_{4}$ gives about $7 \mathrm{~dB}$ gain over the use of an 8 -PSK $\mathcal{G}_{2}$ code.

In Figs. 4 and 5, we provide bit and symbol error rates, respectively, for transmission of $2 \mathrm{bits} / \mathrm{s} / \mathrm{Hz}$ using two, three, and four transmit antennas (also an uncoded 4-PSK). The transmission using two transmit antennas employs the 4-PSK constellation and the code $\mathcal{G}_{2}$. For three and four transmit antennas, the 16-QAM constellation and the codes $\mathcal{G}_{3}$ and $\mathcal{G}_{4}$, respectively, are used. Since $\mathcal{G}_{3}$ and $\mathcal{G}_{4}$ are rate $1 / 2$ codes, the total transmission rate in each case is $2 \mathrm{bits} / \mathrm{s} / \mathrm{Hz}$. It is seen that at the bit error rate of $10^{-5}$ the rate $1 / 216-\mathrm{QAM}$ code $\mathcal{G}_{4}$ gives about $5 \mathrm{~dB}$ gain over the use of a 4-PSK $\mathcal{G}_{2}$ code. 


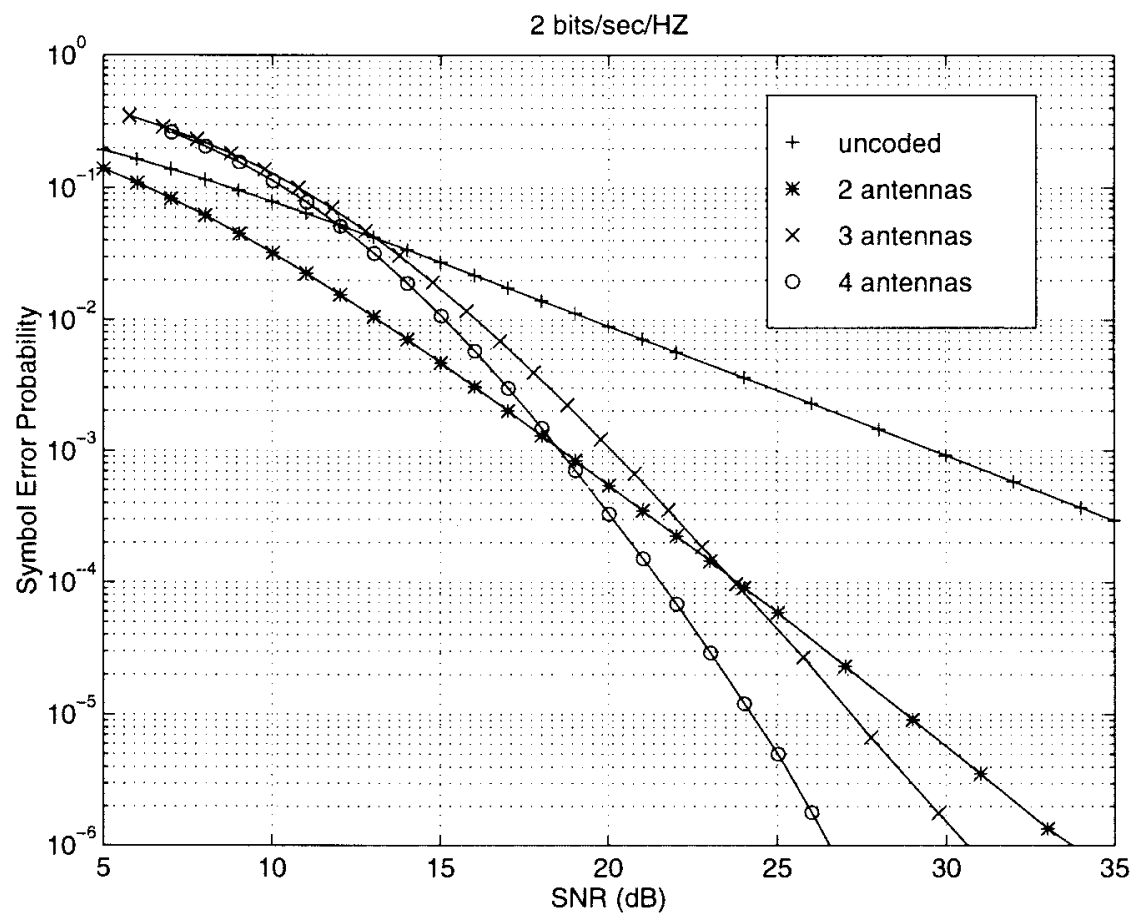

Fig. 5. Symbol error probability versus SNR for space-time block codes at 2 bits/s/Hz; one receive antenna.

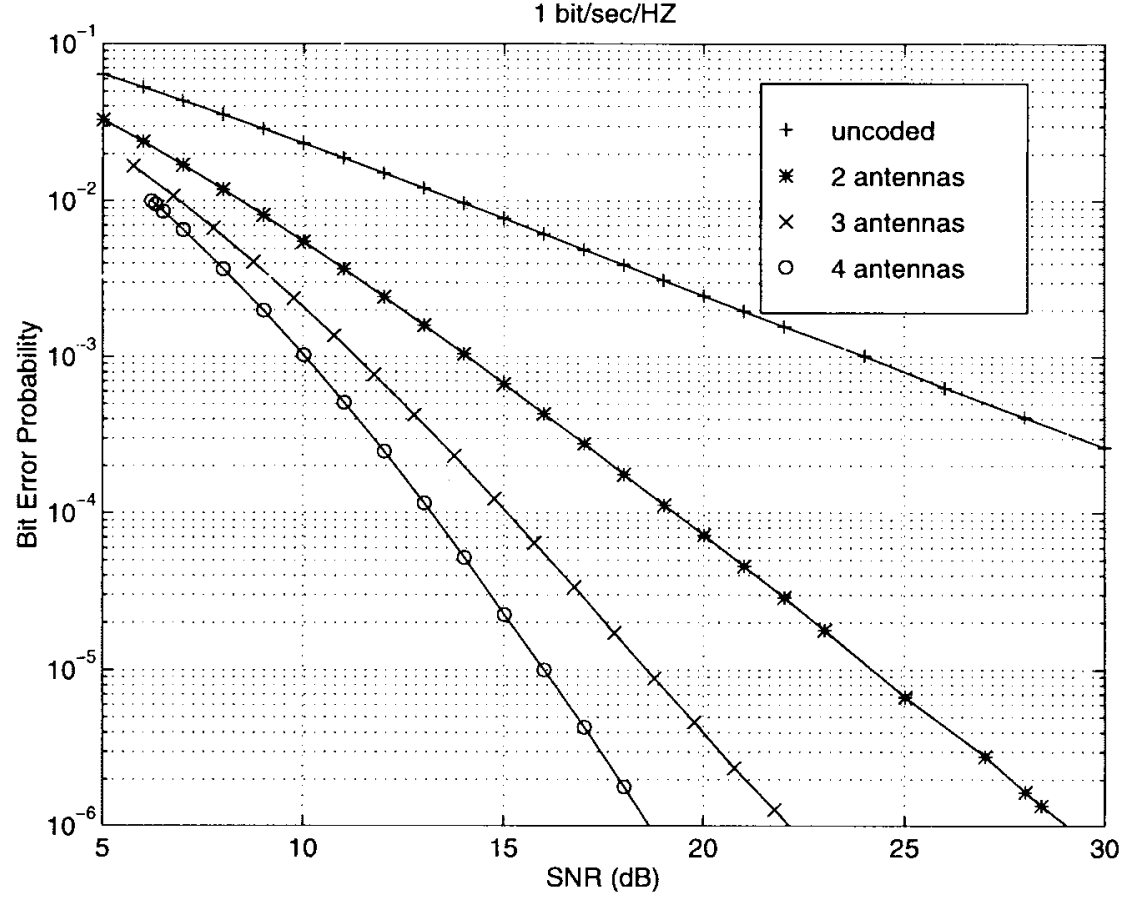

Fig. 6. Bit error probability versus SNR for space-time block codes at $1 \mathrm{bit} / \mathrm{s} / \mathrm{Hz}$; one receive antenna.

Figs. 6 and 7 provide simulation results for transmission of $1 \mathrm{bit} / \mathrm{s} / \mathrm{Hz}$ using one (uncoded), two, three, and four transmit antennas (bit and symbol error rates, respectively). The transmission using two transmit antennas employs the binary PSK (BPSK) constellation and the code $\mathcal{G}_{2}$. For three and four transmit antennas, the 4-PSK constellation and the codes $\mathcal{G}_{3}$ and $\mathcal{G}_{4}$, respectively, are used. Since $\mathcal{G}_{3}$ and $\mathcal{G}_{4}$ are rate $1 / 2$ codes, the total transmission rate in each case is $1 \mathrm{bit} / \mathrm{s} / \mathrm{Hz}$. It is seen that at the bit error rate of $10^{-5}$ the rate $1 / 2$ 4-PSK code $\mathcal{G}_{4}$ gives about $7.5 \mathrm{~dB}$ gain over the use of a BPSK $\mathcal{G}_{2}$ code. If the number of the receive antennas is increased, this gain reduces to $3.5 \mathrm{~dB}$ (Figs. 8 and 9). The reason is that much of the diversity gain is already achieved using two transmit and two receive antennas.

The above simulations demonstrate that significant gains can be achieved by increasing the number of transmit antennas with very little decoding complexity. 


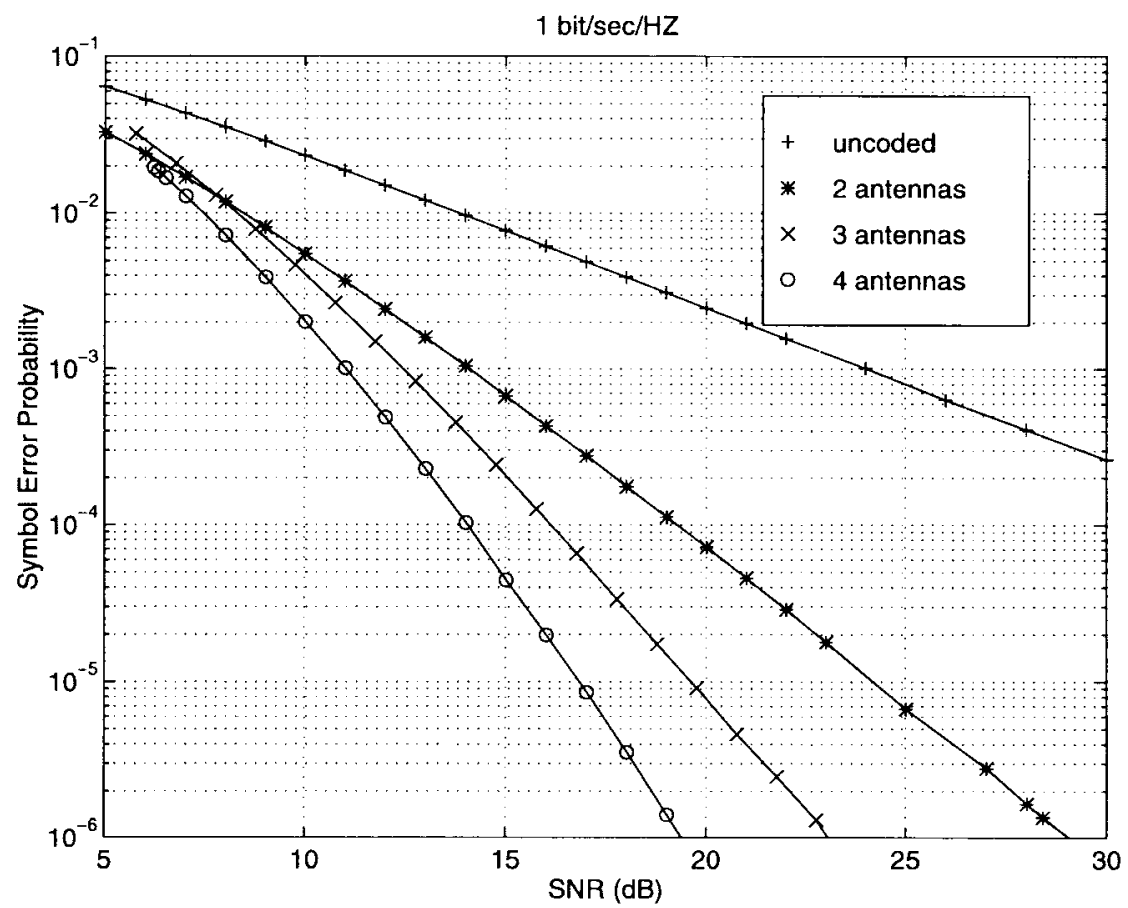

Fig. 7. Symbol error probability versus SNR for space-time block codes at $1 \mathrm{bit} / \mathrm{s} / \mathrm{Hz}$; one receive antenna.

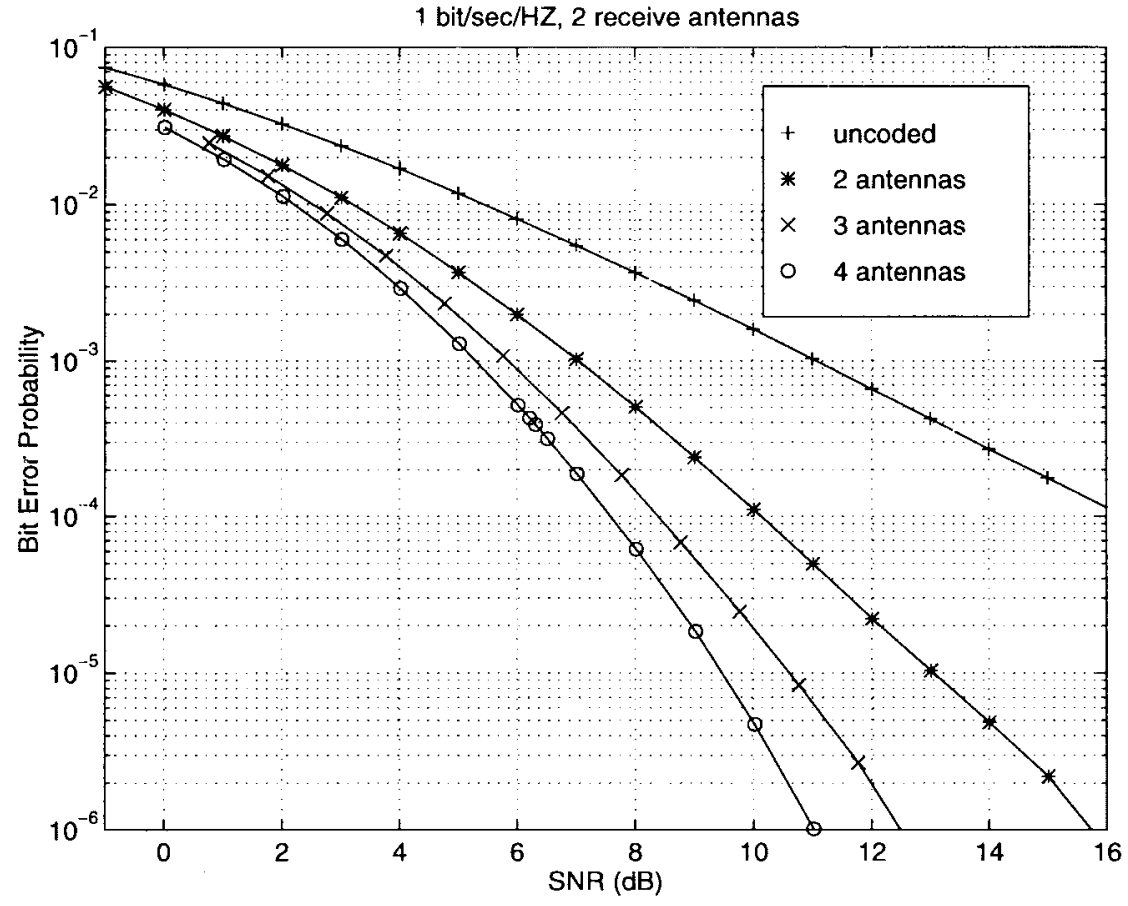

Fig. 8. Bit error probability versus SNR for space-time block codes at $1 \mathrm{bit} / \mathrm{s} / \mathrm{Hz}$; two receive antennas.

It is possible to concatenate an outer trellis code [2] with the above space-time block coding to achieve even better performance. The additional coding gain provided by the outer code is the same as the gain provided by that code on a Gaussian channel. The decision metrics given in this paper for the inner space-time block code then can be used as the branch metrics for the outer trellis code [2]. This gives better performance at the expense of a higher complexity.

\section{CONCLUSION}

We provided examples of space-time block codes for transmission using multiple transmit antennas. We described both their encoding and decoding algorithms. The encoding and decoding of these codes have very little complexity. Simulation results were provided to demonstrate that significant gains can be achieved by increasing the number of transmit chains with very little decoding complexity. 


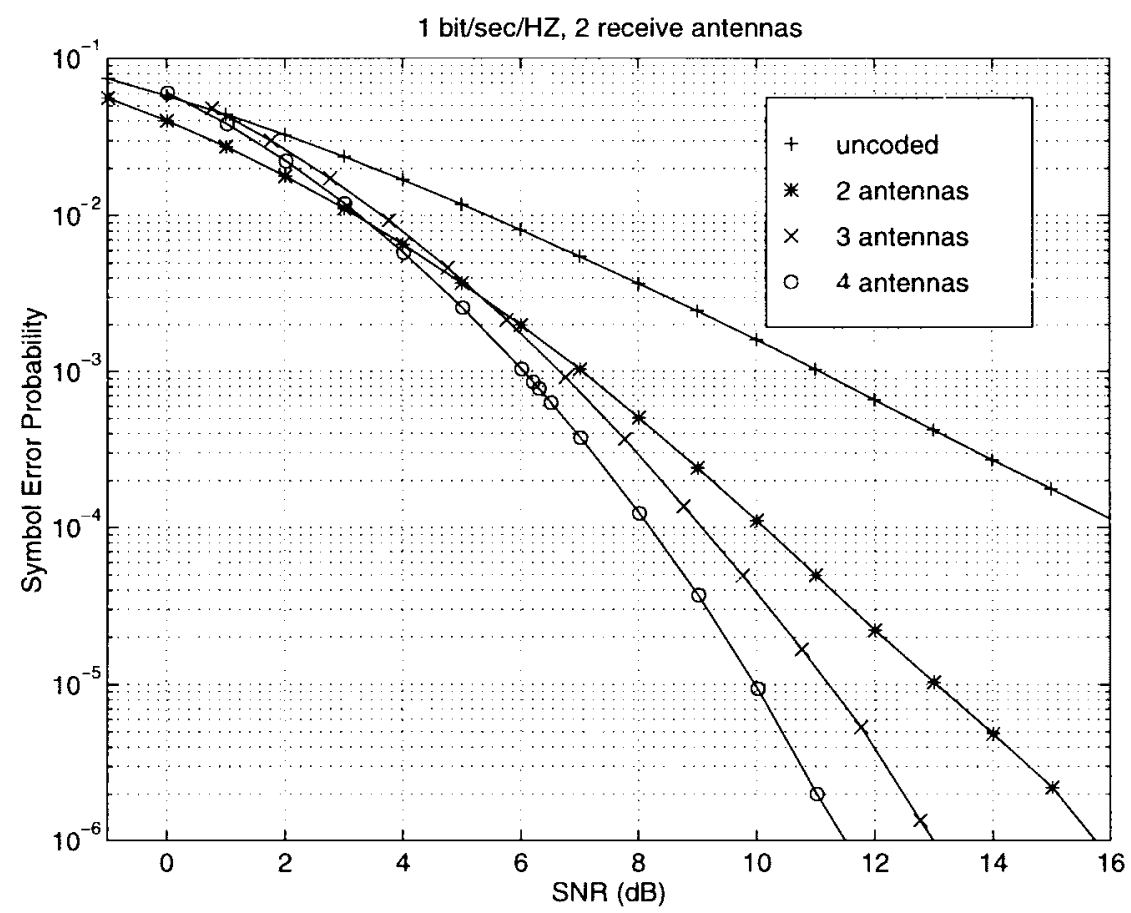

Fig. 9. Symbol error probability versus SNR for space-time block codes at $1 \mathrm{bit} / \mathrm{s} / \mathrm{Hz}$; two receive antennas.

\section{APPENDIX}

In this appendix, we provide specific formulas for decoding $\mathcal{G}_{3}, \mathcal{G}_{4}, \mathcal{H}_{3}$, and $\mathcal{H}_{4}$.

The decoder for $\mathcal{G}_{3}$ minimizes the decision metric

$$
\begin{gathered}
\mid\left[\sum _ { j = 1 } ^ { m } \left(r_{1}^{j} \alpha_{1, j}^{*}+r_{2}^{j} \alpha_{2, j}^{*}+r_{3}^{j} \alpha_{3, j}^{*}+\left(r_{5}^{j}\right)^{*} \alpha_{1, j}\right.\right. \\
\left.\left.\quad+\left(r_{6}^{j}\right)^{*} \alpha_{2, j}+\left(r_{7}^{j}\right)^{*} \alpha_{3, j}\right)\right]-\left.s_{1}\right|^{2} \\
\quad+\left(-1+2 \sum_{j=1}^{m} \sum_{i=1}^{3}\left|\alpha_{i, j}\right|^{2}\right)\left|s_{1}\right|^{2}
\end{gathered}
$$

for decoding $s_{1}$, the decision metric

$$
\begin{gathered}
\mid\left[\sum _ { j = 1 } ^ { m } \left(r_{1}^{j} \alpha_{2, j}^{*}-r_{2}^{j} \alpha_{1, j}^{*}+r_{4}^{j} \alpha_{3, j}^{*}+\left(r_{5}^{j}\right)^{*} \alpha_{2, j}\right.\right. \\
\left.\left.\quad-\left(r_{6}^{j}\right)^{*} \alpha_{1, j}+\left(r_{8}^{j}\right)^{*} \alpha_{3, j}\right)\right]-\left.s_{2}\right|^{2} \\
\quad+\left(-1+2 \sum_{j=1}^{m} \sum_{i=1}^{3}\left|\alpha_{i, j}\right|^{2}\right)\left|s_{2}\right|^{2}
\end{gathered}
$$

for decoding $s_{2}$, the decision metric

$$
\mid\left[\sum _ { j = 1 } ^ { m } \left(r_{1}^{j} \alpha_{3, j}^{*}-r_{3}^{j} \alpha_{1, j}^{*}-r_{4}^{j} \alpha_{2, j}^{*}+\left(r_{5}^{j}\right)^{*} \alpha_{3, j}\right.\right.
$$

$$
\begin{aligned}
& \left.\left.-\left(r_{7}^{j}\right)^{*} \alpha_{1, j}-\left(r_{8}^{j}\right)^{*} \alpha_{2, j}\right)\right]-\left.s_{3}\right|^{2} \\
& +\left(-1+2 \sum_{j=1}^{m} \sum_{i=1}^{3}\left|\alpha_{i, j}\right|^{2}\right)\left|s_{3}\right|^{2}
\end{aligned}
$$

for decoding $s_{3}$, and the decision metric

$$
\begin{aligned}
& \mid\left[\sum _ { j = 1 } ^ { m } \left(-r_{2}^{j} \alpha_{3, j}^{*}+r_{3}^{j} \alpha_{2, j}^{*}-r_{4}^{j} \alpha_{1, j}^{*}-\left(r_{6}^{j}\right)^{*} \alpha_{3, j}\right.\right. \\
& \left.\left.\quad+\left(r_{7}^{j}\right)^{*} \alpha_{2, j}-\left(r_{8}^{j}\right)^{*} \alpha_{1, j}\right)\right]-\left.s_{4}\right|^{2} \\
& \quad+\left(-1+2 \sum_{j=1}^{m} \sum_{i=1}^{3}\left|\alpha_{i, j}\right|^{2}\right)\left|s_{4}\right|^{2}
\end{aligned}
$$

for decoding $s_{4}$.

For decoding $\mathcal{G}_{4}$, the decoder minimizes the decision metric

$$
\begin{aligned}
& \mid\left[\sum _ { j = 1 } ^ { m } \left(r_{1}^{j} \alpha_{1, j}^{*}+r_{2}^{j} \alpha_{2, j}^{*}+r_{3}^{j} \alpha_{3, j}^{*}+r_{4}^{j} \alpha_{4, j}^{*}+\left(r_{5}^{j}\right)^{*} \alpha_{1, j}\right.\right. \\
& \left.\left.\quad+\left(r_{6}^{j}\right)^{*} \alpha_{2, j}+\left(r_{7}^{j}\right)^{*} \alpha_{3, j}+\left(r_{8}^{j}\right)^{*} \alpha_{4, j}\right)\right]-\left.s_{1}\right|^{2} \\
& \quad+\left(-1+2 \sum_{j=1}^{m} \sum_{i=1}^{4}\left|\alpha_{i, j}\right|^{2}\right)\left|s_{1}\right|^{2}
\end{aligned}
$$


for decoding $s_{1}$, the decision metric

$$
\begin{aligned}
& \mid\left[\sum _ { j = 1 } ^ { m } \left(r_{1}^{j} \alpha_{2, j}^{*}-r_{2}^{j} \alpha_{1, j}^{*}-r_{3}^{j} \alpha_{4, j}^{*}+r_{4}^{j} \alpha_{3, j}^{*}+\left(r_{5}^{j}\right)^{*} \alpha_{2, j}\right.\right. \\
& \left.\left.\quad-\left(r_{6}^{j}\right)^{*} \alpha_{1, j}-\left(r_{7}^{j}\right)^{*} \alpha_{4, j}+\left(r_{8}^{j}\right)^{*} \alpha_{3, j}\right)\right]-\left.s_{2}\right|^{2} \\
& \quad+\left(-1+2 \sum_{j=1}^{m} \sum_{i=1}^{4}\left|\alpha_{i, j}\right|^{2}\right)\left|s_{2}\right|^{2}
\end{aligned}
$$

for decoding $s_{2}$, the decision metric

$$
\begin{aligned}
& \mid\left[\sum _ { j = 1 } ^ { m } \left(r_{1}^{j} \alpha_{3, j}^{*}+r_{2}^{j} \alpha_{4, j}^{*}-r_{3}^{j} \alpha_{1, j}^{*}-r_{4}^{j} \alpha_{2, j}^{*}+\left(r_{5}^{j}\right)^{*} \alpha_{3, j}\right.\right. \\
& \left.\left.\quad+\left(r_{6}^{j}\right)^{*} \alpha_{4, j}-\left(r_{7}^{j}\right)^{*} \alpha_{1, j}-\left(r_{8}^{j}\right)^{*} \alpha_{2, j}\right)\right]-\left.s_{3}\right|^{2} \\
& \quad+\left(-1+2 \sum_{j=1}^{m} \sum_{i=1}^{4}\left|\alpha_{i, j}\right|^{2}\right)\left|s_{3}\right|^{2}
\end{aligned}
$$

for decoding $s_{3}$, and the decision metric

$$
\begin{aligned}
& \mid\left[\sum _ { j = 1 } ^ { m } \left(-r_{1}^{j} \alpha_{4, j}^{*}-r_{2}^{j} \alpha_{3, j}^{*}+r_{3}^{j} \alpha_{2, j}^{*}-r_{4}^{j} \alpha_{1, j}^{*}-\left(r_{5}^{j}\right)^{*} \alpha_{4, j}\right.\right. \\
& \left.\left.\quad-\left(r_{6}^{j}\right)^{*} \alpha_{3, j}+\left(r_{7}^{j}\right)^{*} \alpha_{2, j}-\left(r_{8}^{j}\right)^{*} \alpha_{1, j}\right)\right]-\left.s_{4}\right|^{2} \\
& \quad+\left(-1+2 \sum_{j=1}^{m} \sum_{i=1}^{4}\left|\alpha_{i, j}\right|^{2}\right)\left|s_{4}\right|^{2}
\end{aligned}
$$

for decoding $s_{4}$.

To decode the rate $3 / 4$ code $\mathcal{H}_{3}$, the decoder minimizes the decision metric

$$
\begin{aligned}
& \mid\left[\sum _ { j = 1 } ^ { m } \left(r_{1}^{j} \alpha_{1, j}^{*}+\left(r_{2}^{j}\right)^{*} \alpha_{2, j}+\frac{\left(r_{4}^{j}-r_{3}^{j}\right) \alpha_{3, j}^{*}}{2}\right.\right. \\
& \left.\left.\quad-\frac{\left.\left(r_{3}^{j}+r_{4}^{j}\right)^{*} \alpha_{3, j}\right)}{2}\right)\right]-\left.s_{1}\right|^{2} \\
& \quad+\left(-1+\sum_{j=1}^{m} \sum_{i=1}^{3}\left|\alpha_{i, j}\right|^{2}\right)\left|s_{1}\right|^{2}
\end{aligned}
$$

for decoding $s_{1}$, the decision metric

$$
\begin{aligned}
& \mid\left[\sum _ { j = 1 } ^ { m } \left(r_{1}^{j} \alpha_{2, j}^{*}-\left(r_{2}^{j}\right)^{*} \alpha_{1, j}+\frac{\left(r_{4}^{j}+r_{3}^{j}\right) \alpha_{3, j}^{*}}{2}\right.\right. \\
& \left.\left.\quad+\frac{\left(-r_{3}^{j}+r_{4}^{j}\right)^{*} \alpha_{3, j}}{2}\right)\right]-\left.s_{2}\right|^{2} \\
& \quad+\left(-1+\sum_{j=1}^{m} \sum_{i=1}^{3}\left|\alpha_{i, j}\right|^{2}\right)\left|s_{2}\right|^{2}
\end{aligned}
$$

for decoding $s_{2}$, and the decision metric

$$
\begin{aligned}
& \mid\left[\sum _ { j = 1 } ^ { m } \left(\frac{\left(r_{1}^{j}+r_{2}^{j}\right) \alpha_{3, j}^{*}}{\sqrt{2}}+\frac{\left(r_{3}^{j}\right)^{*}\left(\alpha_{1, j}+\alpha_{2, j}\right)}{\sqrt{2}}\right.\right. \\
& \left.\left.\quad+\frac{\left(r_{4}^{j}\right)^{*}\left(\alpha_{1, j}-\alpha_{2, j}\right)}{\sqrt{2}}\right)\right]-\left.s_{3}\right|^{2} \\
& \quad+\left(-1+\sum_{j=1}^{m} \sum_{i=1}^{3}\left|\alpha_{i, j}\right|^{2}\right)\left|s_{3}\right|^{2}
\end{aligned}
$$

for decoding $s_{3}$.

Similarly, to decode the rate $3 / 4$ code $\mathcal{H}_{4}$, the decoder minimizes the decision metric

$$
\begin{aligned}
& \mid\left[\sum _ { j = 1 } ^ { m } \left(r_{1}^{j} \alpha_{1, j}^{*}+\left(r_{2}^{j}\right)^{*} \alpha_{2, j}+\frac{\left(r_{4}^{j}-r_{3}^{j}\right)\left(\alpha_{3, j}^{*}-\alpha_{4, j}^{*}\right)}{2}\right.\right. \\
& \left.\left.\quad-\frac{\left(r_{3}^{j}+r_{4}^{j}\right)^{*}\left(\alpha_{3, j}+\alpha_{4, j}\right)}{2}\right)\right]-\left.s_{1}\right|^{2} \\
& \quad+\left(-1+\sum_{j=1}^{m} \sum_{i=1}^{4}\left|\alpha_{i, j}\right|^{2}\right)\left|s_{1}\right|^{2}
\end{aligned}
$$

for decoding $s_{1}$, the decision metric

$$
\begin{aligned}
& \mid\left[\sum _ { j = 1 } ^ { m } \left(r_{1}^{j} \alpha_{2, j}^{*}-\left(r_{2}^{j}\right)^{*} \alpha_{1, j}+\frac{\left(r_{4}^{j}+r_{3}^{j}\right)\left(\alpha_{3, j}^{*}-\alpha_{4, j}^{*}\right)}{2}\right.\right. \\
& \left.\left.\quad+\frac{\left(-r_{3}^{j}+r_{4}^{j}\right)^{*}\left(\alpha_{3, j}+\alpha_{4, j}\right)}{2}\right)\right]-\left.s_{2}\right|^{2} \\
& \quad+\left(-1+\sum_{j=1}^{m} \sum_{i=1}^{4}\left|\alpha_{i, j}\right|^{2}\right)\left|s_{2}\right|^{2}
\end{aligned}
$$

for decoding $s_{2}$, and the decision metric

$$
\begin{aligned}
& \mid\left[\sum _ { j = 1 } ^ { m } \left(\frac{\left(r_{1}^{j}+r_{2}^{j}\right) \alpha_{3, j}^{*}}{\sqrt{2}}+\frac{\left(r_{1}^{j}-r_{2}^{j}\right) \alpha_{4, j}^{*}}{\sqrt{2}}\right.\right. \\
& \left.\left.\quad+\frac{\left(r_{3}^{j}\right)^{*}\left(\alpha_{1, j}+\alpha_{2, j}\right)}{\sqrt{2}}+\frac{\left(r_{4}^{j}\right)^{*}\left(\alpha_{1, j}-\alpha_{2, j}\right)}{\sqrt{2}}\right)\right]-\left.s_{3}\right|^{2} \\
& \quad+\left(-1+\sum_{j=1}^{m} \sum_{i=1}^{4}\left|\alpha_{i, j}\right|^{2}\right)\left|s_{3}\right|^{2}
\end{aligned}
$$

for decoding $s_{3}$.

\section{ACKNOWLEDGMENT}

The authors would like to thank anonymous reviewers for helpful comments to improve the presentation of the paper.

\section{REFERENCES}

[1] S. M. Alamouti, "A simple transmitter diversity scheme for wireless communications," IEEE J. Select. Areas Commun., vol. 16, pp. 1451-1458, Oct. 1998. 
[2] S. M. Alamouti, V. Tarokh, and P. Poon, "Trellis coded modulation and transmit diversity: Design criteria and performance evaluation," in Proc. IEEE ICUPC 98, Oct. 1998, pp. 703-707.

[3] N. Balaban and J. Salz, "Dual diversity combining and equalization in digital cellular mobile radio," IEEE Trans. Veh. Technol., vol. 40, pp. 342-354, May 1991.

[4] G. J. Foschini, Jr., "Layered space-time architecture for wireless communication in a fading environment when using multi-element antennas," Bell Labs Tech. J., pp. 41-59, Autumn 1996.

[5] G. J. Foschini, Jr. and M. J. Gans, "On limits of wireless communication in a fading environment when using multiple antennas," Wireless Personal Commun., vol. 6, pp. 311-335, 1998.

[6] J.-C. Guey, M. P. Fitz, M. R. Bell, and W.-Y. Kuo, "Signal design for transmitter diversity wireless communication systems over Rayleigh fading channels," in Proc. IEEE VTC'96, Apr. 1996, pp. 136-140.

[7] A. Hiroike, F. Adachi, and N. Nakajima, "Combined effects of phase sweeping transmitter diversity and channel coding," IEEE Trans. Veh. Technol., vol. 41, pp. 170-176, May 1992.

[8] G. J. Pottie, "System design issues in personal communications," IEEE Personal Commun. Mag., vol. 2, no. 5, pp. 50-67, Oct. 1995.

[9] G. Raleigh and J. M. Cioffi, "Spatio-temporal coding for wireless communications," in Proc. IEEE GLOBECOM'96, Nov. 1996, pp. $1809-1814$

[10] N. Seshadri and J. H. Winters, "Two signaling schemes for improving the error performance of frequency-division-duplex (FDD) transmission systems using transmitter antenna diversity," Int. J. Wireless Inform. Networks, vol. 1, no. 1, 1994

[11] C.-E. W. Sundberg and N. Seshadri, "Coded modulation for fading channels: An overview," invited paper, European Trans. Telecommun. Related Technol., pp. 309-324, May 1993.

[12] V. Tarokh, N. Seshadri, and A. R. Calderbank, "Space-time codes for high data rate wireless communication: Performance analysis and code construction," IEEE Trans. Inform. Theory, vol. 44, no. 2, pp. 744-765, Mar. 1998.

[13] V. Tarokh, H. Jafarkhani, and A. R. Calderbank, "Space-time block codes from orthogonal designs," IEEE Trans. Inform. Theory, to be published.

[14] _ "The application of orthogonal designs to wireless communication," in Proc. IEEE Information Theory Workshop, Killarney, Ireland, June 1998, pp. 46-47.

[15] E. Telatar, "Capacity of multi-antenna Gaussian channels," AT\&T-Bell Laboratories, Internal Tech. Memo., June 1995.

[16] V. Weerackody, "Diversity for direct-sequence spread spectrum system using multiple transmit antennas," in Proc. IEEE ICC'93, May 1993 , pp. $1775-1779$.

[17] J. Winters, J. Salz, and R. D. Gitlin, "The impact of antenna diversity on the capacity of wireless communication systems," IEEE Trans. Commun., vol. 42. no. 2/3/4, pp. 1740-1751, Feb./Mar./Apr. 1994.

[18] A. Wittneben, "Base station modulation diversity for digital SIMULCAST," in Proc. IEEE VTC, May 1993, pp. 505-511.

[19] _ "A new bandwidth efficient transmit antenna modulation diversity scheme for linear digital modulation," in Proc. IEEE ICC'93, May 1993, pp. 1630-1634.

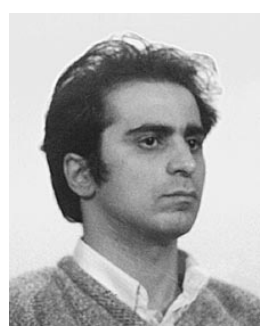

Vahid Tarokh (M'97) received the Ph.D. degree in electrical engineering from the University of Waterloo, Ontario, Canada, in 1995.

$\mathrm{He}$ is currently with AT\&T Laboratories in Florham Park, NJ, as a Senior Technical Staff Member.

Dr. Tarokh was awarded the Gold Medal of the Governor General of Canada for his Ph.D. dissertation.

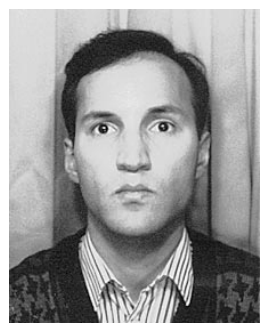

Hamid Jafarkhani (M'86) was born in Tehran, Iran, in 1966. He received the B.S. degree in electronics from Tehran University in 1989 and the M.S and $\mathrm{Ph} . \mathrm{D}$. degrees, both in electrical engineering, from the University of Maryland at College Park in 1994 and 1997, respectively.

Since August 1997, he has been with AT\&T Labs, Red Bank, NJ, as a Senior Technical Staff Member. From June to September 1996, he was a member of the technical staff at Lucent Technologies (Bell Labs).

Dr. Jafarkhani ranked first in the nationwide entrance examination of Iranian universities in 1984. He received University of Maryland graduate school fellowships in 1993 and 1994 and a Texas Instruments DSP fellowship in 1996. He was a corecipient of the American Division Award of the 1995 Texas Instruments DSP Solutions Challenge.

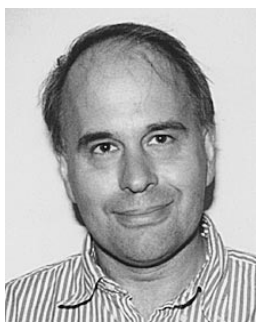

A. Robert Calderbank (M'89-SM'97-F'98) received the B.S. degree in 1975 from Warwick University, England, the M.S. degree in 1976 from Oxford University, England, and the Ph.D. degree in 1980 from the California Institute of Technology, Pasadena, all in mathematics.

$\mathrm{He}$ is Director of the Information Sciences Research Center at AT\&T Labs-Research in Florham Park, NJ. He joined AT\&T Bell Laboratories in 1980, and prior to the split of AT\&T and Lucent, he was a Department Head in the Mathematical Sciences Research Center at Murray Hill. His research interests range from algebraic coding theory to wireline data transmission to quantum computing to wireless communication. At the University of Michigan and at Princeton University, he has developed and taught an innovative course on bandwidth efficient communication.

Dr. Calderbank served on the Information Theory Society Board of Governors from 1990 to 1996 and was Editor-in-Chief of the IEEE TRANSACTIONS ON INFORMation TheOry. He received the 1995 Prize Paper Award from the Information Theory Society for his work on the $Z_{4}$ linearity of the Kerdock and Preparata codes. 